\title{
New Approaches for Carbon Nanotubes-Based Biosensors and Their Application to Cell Culture Monitoring
}

\author{
Cristina Boero, Jacopo Olivo, Giovanni De Micheli, Fellow, IEEE, and Sandro Carrara
}

\begin{abstract}
Amperometric biosensors are complex systems and they require a combination of technologies for their development. The aim of the present work is to propose a new approach in order to develop nanostructured biosensors for the real-time detection of multiple metabolites in cell culture flasks. The fabrication of five Au working electrodes onto silicon substrate is achieved with CMOS compatible microtechnology. Each working electrode presents an area of $0.25 \mathrm{~mm}^{2}$, so structuration with carbon nanotubes and specific functionalization are carried out by using spotting technology, originally developed for microarrays and DNA printing. The electrodes are characterized by cyclic voltammetry and compared with commercially available screen-printed electrodes. Measurements are carried out under flow conditions, so a simple fluidic system is developed to guarantee a continuous flow next to the electrodes. The working electrodes are functionalized with different enzymes and calibrated for the real-time detection of glucose, lactate, and glutamate. Finally, some tests are performed on surnatant conditioned medium sampled from neuroblastoma cells (NG-108 cell line) to detect glucose and lactate concentration after $\mathbf{7 2}$ hours of cultivation. The developed biosensor for real-time and online detection of multiple metabolites shows very promising results towards circuits and systems for cell culture monitoring.
\end{abstract}

Index Terms-Amperometric sensors, biomarkers, carbon nanotubes, cells, electrochemical device.

\section{INTRODUCTION}

$\mathbf{E}$ LECTROCHEMICAL biosensors are complex systems and consist of heterogeneous components: the electrochemical cell, typically with three electrodes; the biological recognition element; the electronics for carrying out measurements; the fluidics, especially for real-time detection. Moreover, many works [1], [2] have recently shown that nanomaterials own very interesting features to enhance sensor performance, especially in the case of biosensors. Thereby, conventional techniques sometimes are not suitable to handle the heterogeneous blocks of a biosensor, especially when nanomaterials are also involved. System miniaturization is another issue to

Manuscript received June 18, 2012; revised September 14, 2012; accepted September 15, 2012. Date of publication November 16, 2012; date of current version November 28, 2012. This work was supported by the NanoTera.ch project I-needle, evaluated by the Swiss National Science Foundation. This paper was recommended by Associate Editor M. Ogorzalek.

The authors are with Integrated Systems Laboratory, Ècole Polytechnique Fédérale de Lausanne, 1015 Lausanne, Switzerland (e-mail: cristina.boero@epfl.ch; jacopo.olivo@epfl.ch; giovanni.demicheli@epfl.ch; sandro.carrara@epfl.ch).

Color versions of one or more of the figures in this paper are available online at http://ieeexplore.ieee.org.

Digital Object Identifier 10.1109/TBCAS.2012.2220137 consider towards the development of integrated systems, so appropriate tools are required to combine such different technologies together. The microfabrication of electrodes can be performed with CMOS compatible technologies, which permits to integrate in the same chip both electrodes and electronics, and shrink everything down to the micro-scale. Electrodes can be made of gold, platinum, or silver by evaporation onto silicon substrate. Afterwards, the sensing element has to be placed on the working electrode. The sensing element for biosensors is typically a protein, which reacts with the target molecule. The straightforward way to deposit protein on the electrodes is by drop casting with pipettes. However, the technique is no longer suitable when electrodes are miniaturized. Thus, we borrow the spotting technology from the field of microarrays and DNA printing. Spotting tools enable to handle ultra-low liquid volume and precisely place drops of $50 \mu \mathrm{m}$ in diameter. Spotting can also help in modifying the electrode surface, for example by placing nanomaterials. Carbon nanotubes (CNT) have shown suitable properties for biosensing [3] and they can be used to this purpose. They have to be in a well-dispersed solution for the spotting, and in a non-volatile solvent, since the spotted volume can be down to $500 \mathrm{pl}$. Real-time monitoring requires fresh solution at the electrode surface and equal concentration between the bulk and the interface: for all these reasons the fluidic system plays a central role. Hence, it is also necessary to integrate a fluidic cell next to the electrodes to create a miniaturized electrochemical cell. Channels and chamber can be manufactured in different materials: to this purpose the most used in literature is PDMS (polydimethylsiloxane), which is biocompatible and presents several advantages, like polymerization at room temperature [4] and the possibility to be attached reversibly or irreversibly onto silicon-based materials by oxygen plasma [5]. However, it is also possible to use more rigid materials, like methacrylate combined with o-rings to ensure hermetic enclosure [6].

All the aforementioned steps need to be combined together. The aim of the present paper is to propose existing technologies for the development of fully integrated biosensors for cell monitoring. The final goal of our research is to achieve the detection of multiple metabolites with a single platform that integrates electrodes, electronics and fluidics for the online and real-time detection of such compounds in cell culture medium.

\section{Metabolite Detection in Cell Culture Medium}

Cell analysis demands new tools dedicated to the monitoring of metabolites involved in cell growth. A better understanding 
of the metabolic variation in cell culture can reveal unknown mechanisms implied in several diseases. Pharmaceutic research is also interested in following metabolic processes to estimate drug efficiency tested in vitro [7]. A further example can be found in regenerative medicine with stem cells: the poor knowledge about differentiation mechanisms requires novel technologies to monitor stem cell differentiation to optimize their implant in vivo [8].

Among all the endogenous compounds involved in cell cycle, glucose and lactate play an important role. Cell cultures metabolize mostly glucose via the glycolytic pathway and they produce lactate, which is toxic to animal cells and inhibits cellular metabolism. For this reason, monitoring glucose consumption enables a more efficient glucose metabolism, while limiting lactate accumulation in culture medium [9]. Moreover, glucose consumption is a key indicator of cell activity and can be correlated to growth rate, cell proliferation or differentiation, and cell population dynamics [10]. In our previous work [11], we have already shown that it is possible to detect such metabolites off-line during different cell cycles. When dealing with neural cell lines, another important compound to take into account is glutamate, the most important excitatory neurotransmitter in the mammalian central nervous system. Its monitoring can be fundamental for the investigation of neurological disorders [12]. Moreover, glutamate is also strictly correlated with glutamine, an important energy and nitrogen source present in commercially available media [13]. There are other several interesting compounds to be monitored in cell cultures, like ethanol, 1-amino acids, galactose, etc [14]. To date, the majority of research on this topic is focused on the development of integrated chips where cells can be inoculated and cultivated [15], [16]. On the other hand, minimizing interferences with cells is much more desirable. Few works [17], [18] deal with multiple detection of metabolites in cell cultures and none of them has shown the integration of multiple and nanostructured working electrodes sharing common counter and reference electrodes. CITSens Bio sensors (C-CIT, Switzerland) are the only device in the market able to perform real-time cell monitoring, even if they do not allow multiple detection on the same platform.

In the present research, we use oxidases as sensing element. This particular protein family generates hydrogen peroxide $\left(\mathrm{H}_{2} \mathrm{O}_{2}\right)$ when reacting with the target molecule. The use of this recognition element permits to achieve the monitoring of different targets with the same detection principles. In fact, once the oxidase reacts with its specific substrate, hydrogen peroxide is produced. The electrical detection occurs because $\mathrm{H}_{2} \mathrm{O}_{2}$ is an electrochemical active species which oxidates when a DC potential of $+650 \mathrm{mV}$ is applied. Therefore, the current intensity is proportional to the metabolite concentration reacting with the enzyme. Such measurements are typically done by a potentiostat instrument. The main steps for biosensor fabrication and potentiostat design are considered in the following sections.

\section{Microfabrication AND FunCtionALiZATION}

The detection of multiple targets requires multiple working electrodes (WEs). We modify WEs by using carbon nanotubes

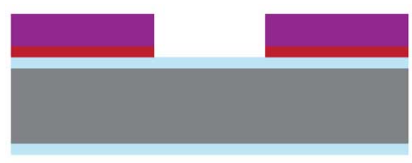

(a)

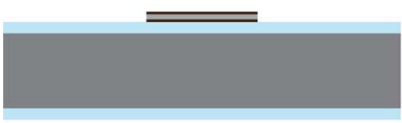

(c)

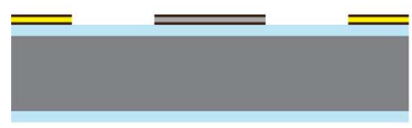

(e)

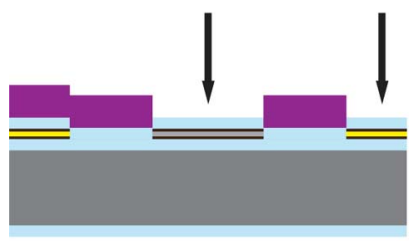

(g)

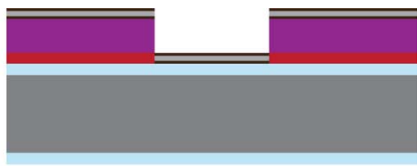

(b)

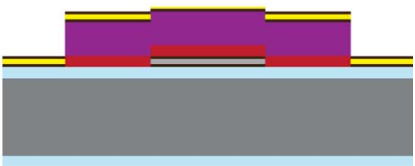

(d)

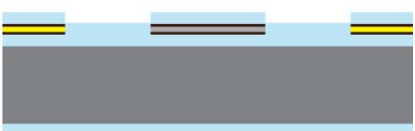

(f)

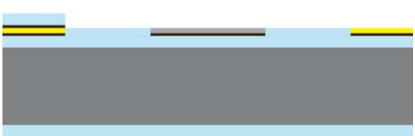

(h)
Fig. 1. Process flow for the microfabrication of the electrochemical cell with multiple WEs. (a) Photoresist (PR) deposition for Pt electrode patterning. (b) $\mathrm{Cr} / \mathrm{Pt} / \mathrm{Cr}$ deposition by PVD. (c) Lift-off of the $\mathrm{Cr} / \mathrm{Pt} / \mathrm{Cr}$. (d) $\mathrm{PR}$ deposition for Au electrode patterning. (e) Lift-off of the $\mathrm{Cr} / \mathrm{Au} / \mathrm{Cr}$. (f) $\mathrm{SiO}_{2} \mathrm{RF}$ sputtering. (g) PR deposition and etching of the $\mathrm{SiO}_{2}$ and $\mathrm{Cr}$ layer. (h) PR stripping.

(CNT), since they have specific electronic properties, like good conductivity [19], high electron transfer rate [14], and considerable electronic emission [20]. These properties result in enhanced sensitivities and lower detection limit from the point-ofview of the biosensor [2], [3]. Then, different protein functionalization of WEs will guarantee the multiple detection of metabolites.

\section{A. Microfabrication}

The chip is conceived with five working electrodes, in order to detect up to five different metabolites at the same time. It consists of gold WEs, whose surface will be modified by CNT afterwards, gold counter electrode, and platinum reference electrode. A schematic of the microfabrication process is illustrated in Fig. 1. Metal layers are evaporated by physical vapor deposition (PVD) onto $525 \mu \mathrm{m}\langle 100\rangle$ silicon substrate covered by $500 \mathrm{~nm}$ of $\mathrm{SiO}_{2}$. Au and Pt thin films $(200 \mathrm{~nm})$ are patterned to form the electrodes and connections by photolithography and lift-off processes. A thin film of $\mathrm{Cr}(20 \mathrm{~nm})$ is deposited on top and under the metal layer, as adhesion layer for metals and passivation. All the WEs are identical and they have an area of $0.25 \mathrm{~mm}^{2}$. Connector insulation is achieved by radio-frequency (RF) sputtering of $\mathrm{SiO}_{2}$ and successive dry etching by deep reactive ion-etching (DRIE) of the $\mathrm{SiO}_{2}$ and wet etching (mixture of ceric ammonium nitrate and perchloric acid) of $\mathrm{Cr}$ etching next to the electrodes and pads for electronic connections. The interface between the chip and the potentiostat is guaranteed by using a commercial edge card socket, while the switching among the five WEs is achieved by a manual switch in the first prototype. 


\section{B. CNT Spotting}

Multi-walled carbon nanotubes (MWCNT_diameter $10 \mathrm{~nm}$, length $1-2 \mu \mathrm{m})$ are purchased in powder (90\% purity) from Dropsens (Spain). Then, they are dispersed in a solution of distilled water $50 \mathrm{vv} \%$ and ethanol $50 \mathrm{vv} \%$, with $0.5 \mathrm{wt} \%$ on Nafion (purchased from Sigma-Aldrich, Switzerland). Final concentration of MWCNT is $1 \mathrm{mg} / \mathrm{ml}$. The Nafion-based solution is chosen according to the high solubility that carbon nanotubes showed in the research of Wang et al. [21]. It is preferred to dispersion in chloroform because it is too volatile for the volumes involved in our study. Automatic spotting of CNT is performed with a commercially available non-contact spotter (sciFLEXARRAYER DW by Scienion, Germany). The minimum dispensed volume is around $400 \mathrm{pl}$ and can be increased by increasing the number of drops per spot. According to previous results obtained with macroelectrodes [22], we deposit $600 \mathrm{ng}$ of CNT, corresponding to a volume of $600 \mathrm{nl}$ of MWCNT/Nafion solution. Drop size is around 50-80 $\mu \mathrm{m}$ with the tendency to aggregate. This is due to the fact that walls of carbon nanotubes are highly hydrophobic. Therefore, we pattern the electrode area as a matrix of $5 \times 5$ spots, to cover the entire electrode. Then, we dispense 10 drops for each spot (around $4 \mathrm{nl}$ per spot) for 5 cycles. The dimension and the volume of the drops can be monitored at the end of each spotting by using a camera installed on the instrument. After the Nafion solution is dry, the carbon nanotubes uniformly cover the electrode surface.

\section{Functionalization With Enzymatic Probes}

In the present research we show results related to glucose, lactate, and glutamate detection. However, the same principles can be used for other metabolite detections. Glucose oxidase from Aspergillus Niger (GOD, EC 1.1.3.4, 129.9 units/mg solid), glutaraldehyde solution (25\% water), D-(+)-glucose, lithium L-lactate, and L-glutamic acid were purchased from Sigma-Aldrich (Switzerland) as lyophilized powders. Glutamate oxidase from Streptomyces species (GlOD, EC 1.4.3.11, 25 units) was supplied from Yamasa Co. (Japan), while lactate oxidase from Pediococcus species (LOD, EC 1.13.12.4, $\geq 20$ units/mg solid) was purchased from Roche Diagnostics GmbH (Germany). All the enzymes are dissolved in Phosphate Buffer Saline solution (PBS) $0.01 \mathrm{M}$ at $\mathrm{pH} 7.4$ and glutaraldehyde $0.25 \%$ (GOD $50 \mathrm{mg} / \mathrm{ml}$, LOD $66 \mathrm{mg} / \mathrm{ml}$, and GlOD $0.6 \mathrm{U} / \mathrm{ml}$ ), while metabolites are dissolved in PBS solution or cell culture medium. For GOD and LOD the enzyme concentration is enough to spot 100 drops for each electrode $(5 \times 5$ matrix, 4 drops per spot), while GlOD is more diluted, so we need 1000 drops onto the electrode area $(10 \times 10$ matrix, 10 drops per spot). Once the enzymes are dropped on the electrode, the proteins are adsorbed onto carbon nanotube walls, as previously discussed in [11].

\section{Cell Culture}

NG-108 neuroblastoma cells belong to a neuronal cell line which exhibits two different morphologies according to the cell state. They are supplied by Prof. Horst Vogel (EPFL, Switzerland). Cells are maintained in Dulbecco's modified Eagle's medium (DMEM/F12 + Glutamax, Gibco-Invitrogen, Switzerland), supplemented with 10\% Foetal Bovine

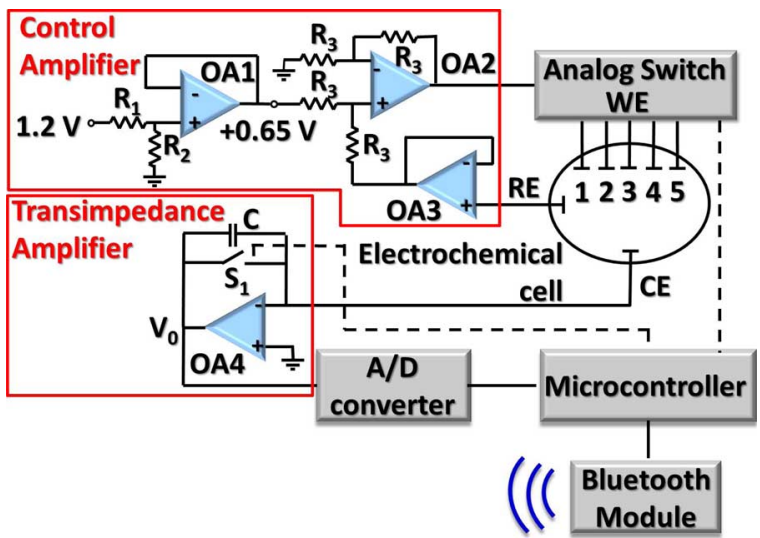

Fig. 2. A schematic of the architecture conceived for the electrochemical measurements of metabolites in cell culture monitoring.

Serum (FBS, Gibco/BRL, Rockville, MD, USA) in $25 \mathrm{~cm}^{2}$ culture flasks (Corning, New York, NY USA) in a $5 \% \mathrm{CO}_{2}$ atmosphere at $37^{\circ} \mathrm{C}$. When reaching $80-90 \%$ of confluence (normally after 48-72 hours of culture), cells are split with $0.05 \%$ Trypsin-EDTA (Gibco-Invitrogen, Switzerland) and seeded in new flasks, while surnatant conditioned medium is collected for glucose and lactate level measurements [23].

\section{Architectural Design}

As previously mentioned, we aim to develop an integrated platform for the monitoring of different metabolites in cell culture. Electrode fabrication was already described in Section III-A. In this Section we discuss the electronics needed to perform electrochemical measurements and we present a possible design for the potentiostat that is currently under development. The use of such biosensors for cell cultures complicates the design, since cells are continuously kept in the incubator and the system has to be as less invasive as possible. A wireless communication between the chip and a remote station is highly desirable, while the user should have the possibility to remotely choose the metabolite to monitor and activate the measurement. The proposed architecture under development is illustrated in Fig. 2.

Electrochemical biosensors require a potentiostat to perform the measurement. Each oxidase is selective to one specific target and hydrogen peroxide is generated by the redox reaction. $\mathrm{H}_{2} \mathrm{O}_{2}$ concentration can be measured by keeping a constant potential of $650 \mathrm{mV}$ between working and reference electrodes, while no current is flowing between the two. Due to the applied potential, $\mathrm{H}_{2} \mathrm{O}_{2}$ is electrolyzed and a current flows through the counter electrode. The current value is proportional to the substrate concentration. The user can remotely choose the metabolite to detect by selecting the appropriate working electrode. The setting is transmitted to the microcontroller by means of the Bluetooth module. The microcontroller drives the analog switch to connect the right working electrode.

The potentiostat is conceived in two blocks: the control amplifier and the transimpedance amplifier. Referring to Fig. 2, the control amplifier keeps a constant voltage of $650 \mathrm{mV}$ between working and reference electrodes. The reference electrode is connected to a voltage follower (OA3); thus, no current 
flows through it. The counter electrode is connected to a transimpedance amplifier (OA4). The virtual short-circuit between the inputs of OA4 forces a virtual ground on the counter electrode (grounded counter configuration). During the measurement, $\mathrm{S}_{1}$ is open and the current flowing through the working electrode charges the capacitor $\mathrm{C}$. The slope of the charging curve $(\Delta \mathrm{V} / \Delta \mathrm{t})$ is directly proportional to the value of the current, accordingly to the expression

$$
I=C \frac{\Delta V}{\Delta t} .
$$

The current $\mathrm{I}$ is assumed to be constant during the whole charge. By properly selecting the value of the capacitor $\mathrm{C}$ and the integration time $\Delta \mathrm{t}$, different ranges of current can be measured. After every measurement, the capacitor is discharged by closing the switch $S_{1}$. The output voltage of the transimpedance amplifier is digitalized and transmitted to a remote station by the Bluetooth module. The whole system is powered by using standard batteries to avoid contamination of the cell culture due to external cables.

\section{OnLine Measurements}

First we need to characterize the bare electrodes and investigate their properties. Due to the lithographic process, we have to check that the active area of different electrodes is similar. To this purpose, we exploit them with cyclic voltammetry (CV), using ferricyanide, which is a well-known and stable species. Afterwards, we compare the recorded $\mathrm{CV}$ with those obtained with commercially available screen-printed electrodes (SPE) (Dropsens, Spain). SPE consists of a gold working electrode (with area of $2 \mathrm{~mm}^{2}$ ), gold counter, and silver reference electrodes. Once the microfabricated electrodes are nanostractured and functionalized, we proceed with their calibration according to the enzyme probe. We finally test the biochip in a cell culture by detecting glucose consumption and lactate production after 72 hours of cultivation. All the electrochemical measurements are acquired by using Autolab potentiostat system (Metrohom, Netherlands).

\section{A. Electrode Characterization}

Ferricyanide solutions are prepared in the range of $0-20 \mathrm{mM}$ in PBS (ferrycianide is purchased in powder from Sigma-Aldrich, Switzerland). For CV characterization, electrodes are kept in quiescent conditions by casting a $100 \mu \mathrm{l}$ drop of substrate to cover the whole electrochemical cell. We perform 10 cycles and we consider the last one, which is the most stable. Microfabricated electrodes are compared with commercially available screen-printed electrodes. We repeat the measurements for several concentrations of ferricyanide for all the five electrodes. Fig. 3 illustrates an example of cyclic voltammetry performed on the five WEs for $10 \mathrm{mM}$ of ferricyanide. The CVs overlap for different electrodes and the two peaks of ferricyanide are well shaped. The average potential for the oxidation peak is $229.4 \pm 7.3 \mathrm{mV}$, while is $109.2 \pm 8.7 \mathrm{mV}$ for reduction peak. Therefore, the difference among different electrodes is lower than $10 \%$ (much lower in the case of oxidation peak). CV is also performed for commercially available screen-printed electrodes (data not shown). Oxidation peak

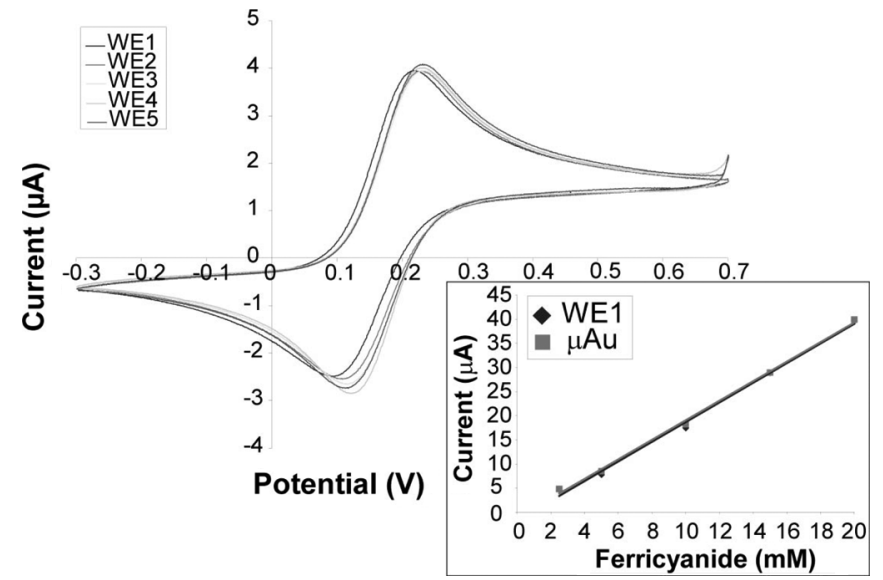

Fig. 3. Cyclic voltammograms of $10 \mathrm{mM}$ ferricyanide for the five working electrodes (scan rate $100 \mathrm{mV} / \mathrm{s}$ ). Inset: comparison between commercial SPE and microfabricated electrodes for ferricyanide.

occurs at $252.7 \pm 27.2 \mathrm{mV}$, with $20 \mathrm{mV}$ shift towards higher potential with respect to the case of microelectrodes. This is due to the reference electrodes, which are made of different materials.

Peak height varies according to substrate concentration for both SPE and microfabricated electrodes. It is possible to compare the two calibration lines, once they are normalized for the electrode area. As shown in the inset of Fig. 3, we notice an almost perfect superimposition of the two lines. Those results guarantee that microfabricated electrodes are suitable to be used as electrochemical biosensors.

\section{B. Calibration in PBS for Different Metabolites}

Calibrations for the different metabolites are performed by means of chronoamperometry under flow conditions with nanostructured and functionalized electrodes. The fluidic system consists of an inner channel, a chamber, and an outlet channel molded in PDMS. The curing agent is mixed with the liquid PDMS in a rate of 1 to 10 and the solution is put in the desiccation chamber for about 1 hour to pull out all the air bubble. Then, the fluid is poured in a PMMA (polymethilmethacrylate) mold and let dry in the oven at $80^{\circ} \mathrm{C}$ for one hour. Once the fluidic system is solidified, it is kept in oxygen plasma for 70 seconds with a power of $20 \mathrm{~W}$ to increase the hydrophilicity before using it. The PDMS chamber is then fixed inside an in-house chip holder made of PMMA to provide easy connections with the rest of the fluidic system (refer to Fig. 4 for a schematic of the biochip and the in-house holder). The inner and outlet channels are connected to a peristaltic pump (Gilson SA, Switzerland) by means of Tygon tubes of $0.25 \mathrm{~mm}$ of internal diameter. The sample solution is circulated with a velocity of around $13 \mu \mathrm{l} / \mathrm{min}$. Once the measurement is run, we change the solution at the inlet of the fluidic circuit around every 3 minutes. A current variation occurs on the active working electrode when the solution is changed. A typical chronoamperometry is depicted in Fig. 5: well-shaped steps are visible every further injection of substrate. Calibration line is calculated from current steps, by measuring the difference between the baseline (related to $0 \mathrm{mM}$ concentration) and the reached value. For 


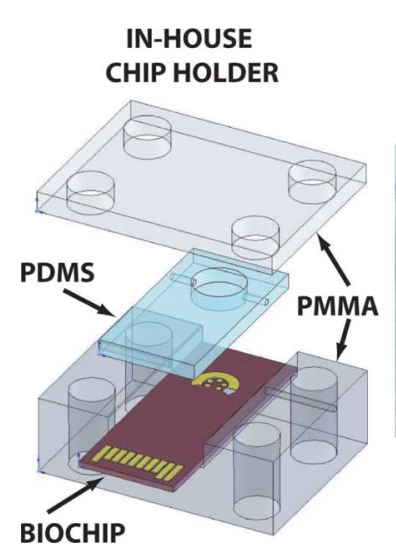

(a)

(b)

Fig. 4. (a) In-house chip holder in PMMA hosting the PDMS chamber and the biochip with multi-working electrodes. (b) A picture of the fluidic system.

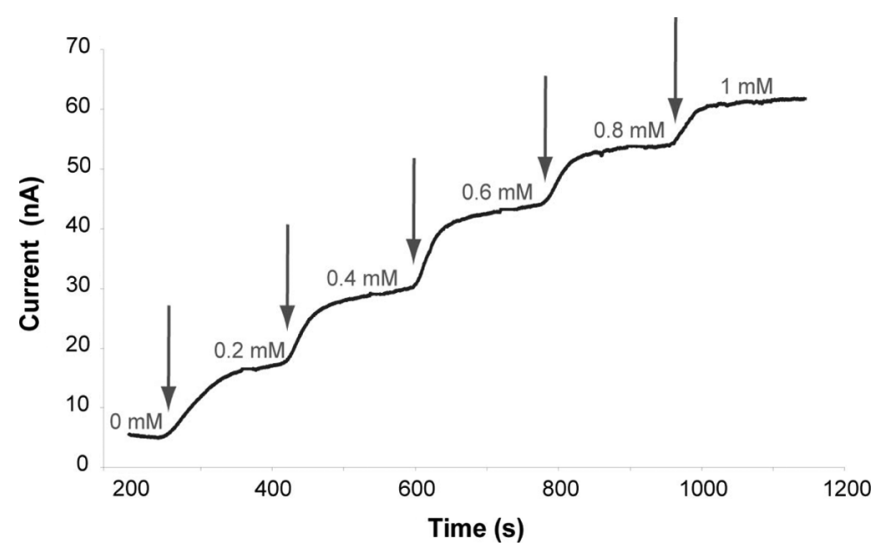

Fig. 5. Typical chronoamperometry for lactate in PBS in the range within 0 and $1 \mathrm{mM}$ (applied potential $+650 \mathrm{mV}$ ). The arrows point out the exchange of solution.

lactate the sensitivity results in a value of $25.0 \mu \mathrm{A} / \mathrm{mM} \mathrm{cm}^{-2}$ and a detection limit of $11 \mu \mathrm{M}\left(\mathrm{S} / \mathrm{N}=3 \sigma, \mathrm{R}^{2}=0.99\right)$. We perform chronoamperometries for glucose and glutamate with the same setup used for lactate (refer to Fig. 6). The sensitivity for glucose is $55.5 \mu \mathrm{A} / \mathrm{mM} \mathrm{cm}^{-2}$ with a detection limit of $2 \mu \mathrm{M}\left(\mathrm{R}^{2}=0.99\right)$, while for glutamate is $0.9 \mu \mathrm{A} / \mathrm{mM} \mathrm{cm}^{-2}$ with a detection limit of $156 \mu \mathrm{M}\left(\mathrm{R}^{2}=0.95\right)$. Glutamate biosensor starts responding from $400 \mu \mathrm{M}$, as shown in the inset of Fig. 6.

\section{Metabolite Detection in NG-108 Cell Line}

Membranes with proper cut-off can significantly extend the linear range of a biosensor playing the role of a diffusion barrier. The drawback is the decrease of the biosensor sensitivity [24]. For these reasons we employ a microdialysis probe as the layer diffusion barrier. The probe is immersed in the culture medium and connected with the fluidic system. The experimental setup appears slightly different for metabolic analysis in cell culture (a schematic is depicted in Fig. 7). Microdialysis probe consists of 2 coaxial channels: the outer wall is a membrane, which pore size is chosen according to molecule size that we want to include in our measurements. A perfusate is flowed through the inner channel (in our case is PBS) and pushed to pass outside at the end of the probe. The solution leaving the probe is enriched by

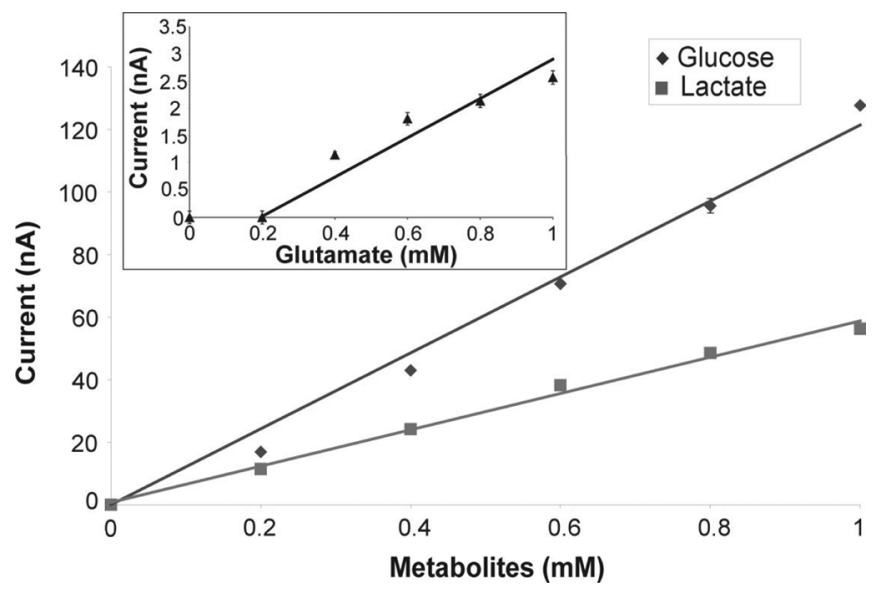

Fig. 6. Calibration lines for the three considered metabolites: glucose (rhombus), lactate (square), and glutamate in the inset (triangles).

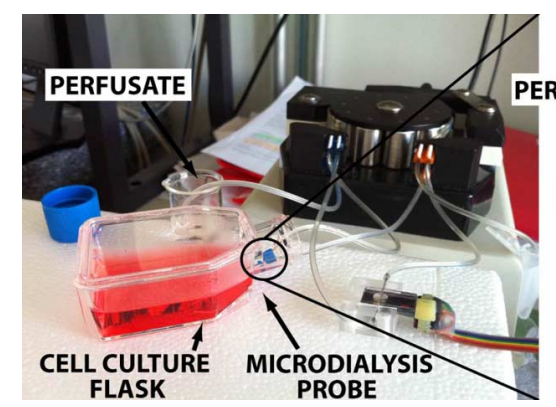

(a)

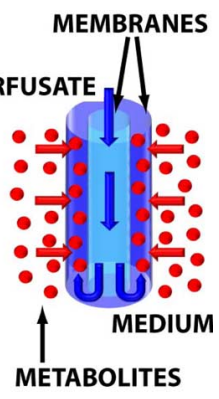

(b)
Fig. 7. (a) Fluidic system for calibration of different metabolites. (b) Working principles for a microdialysis probe.

molecules, which can diffuse through the outer membrane from the medium. A schematic of the probe is illustrated in Fig. 7(b). We use a microdialysis probe (Microbiotech SA, Sweden) with a cut-off of $6 \mathrm{kDa}$. We are interested to detect glucose and lactate for instance, which are small molecules. Glucose has an atomic mass lower than $200 \mathrm{Da}$, while lactate has an atomic mass lower than $100 \mathrm{Da}$. The second advantage of the microdialysis probe is the possibility to be sterilized. With a view to the application in cell culture monitoring, we can avoid contamination even if the probe is in continuous contact with it.

Lactate production and glucose uptake are normal mechanisms in a cell culture. If neuroblastoma cells are in favorable conditions, they keep proliferating. As soon as cell number increases, glucose uptake and lactate production become more important. Surnatant conditioned medium is collected from three different batches of NG-108 cells. We start to detect lactate: the microdialysis probe is dept in fresh DMEM (without lactate) to measure the baseline. Once the measurement is stable, the current value is used as a baseline for the following measurements. The fresh medium is changed with DMEM in which is added lactate (concentration of $10 \mathrm{mM}$ ), without stopping the peristaltic pump and the electrochemical measurement. The continuous monitoring reveals that current increases according to the increase of lactate concentration. The two obtained current values are used for a further check on the calibration of the biosensor. After that, the surnatants coming from the first batch is measured for 5 minutes before the successive sample 


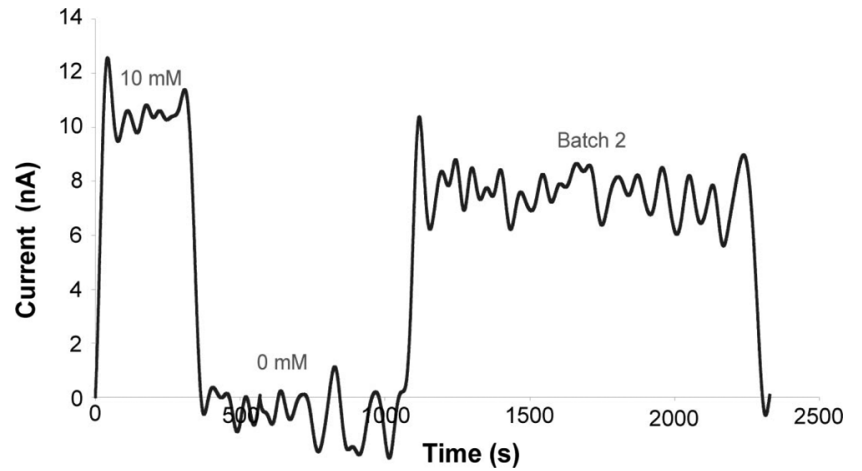

Fig. 8. Measurement of lactate levels in surnatant conditioned medium after 72 hours of NG-108 cell culture. Data are post-processed by using a Butterworth filter.

TABLE I

Lactate Production in SuRnatant Conditioned Medium

\begin{tabular}{l|cc}
\hline Batch & \multicolumn{2}{|c}{ LACTATE } \\
\hline & Day 0 & Day 3 \\
\hline \hline Batch 1 & $0 \mathrm{mM}$ & $10.0 \pm 2.2 \mathrm{mM}$ \\
\hline Batch 2 & $0 \mathrm{mM}$ & $7.80 \pm 2.1 \mathrm{mM}$ \\
\hline Batch 3 & $0 \mathrm{mM}$ & $6.4 \pm 2.0 \mathrm{mM}$ \\
\hline
\end{tabular}

TABLE II

Glucose Uptake in Surnatant Conditioned Medium

\begin{tabular}{l|cc}
\hline Batch & \multicolumn{2}{|c}{ GLUCOSE } \\
\hline & Day 0 & Day 3 \\
\hline \hline Batch 1 & $22.5 \mathrm{mM}$ & $9.2 \pm 1.8 \mathrm{mM}$ \\
\hline
\end{tabular}

to rinse the biochip. The second and the third batches are measured successively. Lactate concentration results in a value of $10.0 \pm 2.2 \mathrm{mM}$ in batch $1,7.8 \pm 2.1 \mathrm{mM}$ in batch 2 , and $6.4 \pm 2.0 \mathrm{mM}$ in batch 3 after 72 hours of cultivation. The measurement related to batch 2 is depicted in Fig. 8, as an example. The detected lactate is quite similar to values reported from Leegsma-Vogt et al. [25] for NG-108 cell line. We perform a similar measurement for glucose in one batch: the baseline and the current related to a nominal value are registered, as in the previous experiment. The calibration occurs referring to the baseline and to a glucose concentration of $22.5 \mathrm{mM}$, which is the concentration normally present in the fresh medium added to cells. The successive measurement, instead, concerns the surnatant after 72 hours of cultivation (data not shown). Glucose uptake can be measured and it results in a value of $9.2 \pm 1.8 \mathrm{mM}$, which is less than half of the initial value of glucose. This is a further confirmation that cells proliferate and this evidence can be fundamental to distinguish different cellular states. Data related to lactate and glucose detection are summarized in Table I and in Table II, respectively.

\section{CONCLUSION}

We described how various heterogeneous technologies can support the development of electrochemical biosensors for the detection of multiple metabolites, such as glucose, lactate and glutamate. We have fabricated a micro-electrochemical cell with five working electrodes. We have shown that spotting tools are suitable for nanostructuring and functionalization, althought they were originally developed for microarrays and DNA printing. We have presented the design under development of the potentiostat for the online monitoring of cell cultures. We have demonstrated that redox reactions on microfabricated electrodes are the same as those obtained with commercially available gold screen-printed electrodes. We achieved the selective functionalization of the electrodes with different oxidases and we calibrated under flow conditions the developed biosensor for glucose, lactate and glutamate detection. We obtained a sensitivity of $25.0 \mu \mathrm{A} / \mathrm{mM} \mathrm{cm}^{-2}$ and a detection limit of $11 \mu \mathrm{M}$ for lactate, a sensitivity of $55.5 \mu \mathrm{A} / \mathrm{mM} \mathrm{cm}^{-2}$ and a detection limit of $2 \mu \mathrm{M}$ for glucose, a sensitivity of $0.9 \mu \mathrm{A} / \mathrm{mM} \mathrm{cm}^{-2}$ and a detection limit of $156 \mu \mathrm{M}$ for glutamate. We tested our carbon nanotubes-based biosensor in a neural cell culture and we detected glucose and lactate in surnatant conditioned medium. Data show lactate production and glucose uptake after 72 hours of cultivation. The present results are really promising towards the online application of such biosensors for the real-time monitoring of multiple metabolites in cell cultures. Few works deal with metabolite detection in cell cultures. However, none of them has shown the integration of multiple and nanostructured WEs sharing common counter and reference electrodes. Instead, data showed in the present paper represent a novelty with respect to the state-of-the-art in cell culture monitoring and pave the way to new integrated systems for cell analysis.

\section{ACKNOWLEDGMENT}

The authors would like to thank all the CMI staff for their support on microfabrication processes. C. Boero and S. Carrara thank Prof. Vogel and Dr. Piguet for help in handling the NG-108 cell line, and Prof. Gijs and Dr. Alramadan for their suggestions about the fluidic system.

\section{REFERENCES}

[1] C. Boero, S. Carrara, and G. De Micheli, "Sensitivity enhancement by carbon nanotubes: Applications to stem cell cultures monitoring," Res. Microelectron. Electron., pp. 72-75, Jul. 2009.

[2] J. J. Gooding, "Nanostructuring electrodes with carbon nanotubes: A review on electrochemistry and applications for sensing," Electrochim. Acta, vol. 50, no. 15, pp. 3049-3060, 2005.

[3] G. A. Rivas, M. D. Rubianes, M. C. Rodríguez, N. F. Ferreyra, G. L. Luque, M. L. Pedano, S. A. Miscoria, and C. Parrado, "Carbon nanotubes for electrochemical biosensing," Talanta, vol. 74, no. 3, pp. 291-307, 2007.

[4] J. C. McDonald and G. M. Whitesides, "Poly(dimethylsiloxane) as a material for fabricating microfluidic devices," Accounts Chem. Res., vol. 35, no. 7, pp. 491-499, 2002.

[5] K. Chau, B. Millare, A. Lin, S. Upadhyayula, V. Nunez, H. Xu, and V. Vullev, "Dependence of the quality of adhesion betweenpoly(dimethylsiloxane) and glass surfaces on the composition of theoxidizing plasma," Microfluid. Nanofluid., vol. 10, pp. 907-917, 2011.

[6] P. Fanjul-Bolado, P. J. Lamas-Ardisana, D. Hernández-Santos, and A. Costa-Garcíia, "Electrochemical study and flow injection analysis of paracetamol in pharmaceutical formulations based on screen-printed electrodes and carbon nanotubes," Anal. Chim. Acta, vol. 638, no. 2, pp. 133-138, 2009.

[7] I. A. Hemmilä and P. Hurskainen, "Novel detection strategies for drug discovery," Drug Discov. Today, vol. 7, no. 18, pp. S150-S156, 2002. 
[8] L. Zhu, G. Del Vecchio, G. De Micheli, Y. Liu, S. Carrara, L. Calzá, and C. Nardini, "Biochips for regenerative medicine: Real-time stemcell continuous monitoring as inferred by high-throughput gene analysis," BioNanoSci., vol. 1, pp. 183-191, 2011.

[9] K. B. Male, P. O. Gartu, A. A. Kamen, and J. H. T. Luong, "On-line monitoring of glucose in mammalian cell culture using a flow injection analysis (fia) mediated biosensor," Biotechnol. Bioeng., vol. 55, no. 3, pp. 497-504, 1997.

[10] F. Zhang, J. Tian, L. Wang, P. He, and Y. Chen, "Correlation between cell growth rate and glucose consumption determined by electrochemical monitoring," Sensor. Actuat. B-Chem., vol. 156, no. 1, pp. 416-422, 2011.

[11] C. Boero, S. Carrara, G. Del Vecchio, L. Calzá, and G. De Micheli, "Targeting of multiple metabolites in neural cells monitored by using protein-based carbon nanotubes," Sensor. Actuat. B-Chem., vol. 157, no. 1, pp. 216-224, 2011

[12] M. A. Rahman, N. Kwon, M. Won, E. S. Choe, and Y. Shim, "Functionalized conducting polymer as an enzyme-immobilizing substrate: An amperometric glutamate microbiosensor for in vivo measurements," Anal. Chem., vol. 77, no. 15, pp. 4854-4860, 2005.

[13] M. Bäcker, L. Delle, A. Poghossian, M. Biselli, W. Zang, P. Wagner, and M. J. Schöning, "Electrochemical sensor array for bioprocess monitoring," Electrochim. Acta, vol. 56, no. 26, pp. 9673-9678, 2011.

[14] V. Vojinovic, F. M. F. Esteves, J. M. S. Cabral, and L. P. Fonseca, "Bienzymatic analytical microreactors for glucose, lactate, ethanol, galactose and l-amino acid monitoring in cell culture media," Anal. Chim. Acta, vol. 565, no. 2, pp. 240-249, 2006.

[15] J. Wiest, M. Schmidhuber, J. Ressler, A. Scholz, M. Brischwein, and B. Wolf, "Cell based assays for diagnostic and therapy on multiparametric biosensor chips with an intelligent mobile lab," IFMBE Proc., vol. 10, pp. 132-135, 2005.

[16] N. Pereira Rodrigues, Y. Sakai, and T. Fujii, "Cell-based microfluidic biochip for the electrochemical real-time monitoring of glucose and oxygen," Sensor. Actuat. B-Chem., vol. 132, no. 2, pp. 608-613, 2008.

[17] J. Perdomo, H. Hinkers, C. Sundermeier, W. Seifert, O. Martínez Morell, and M. Knoll, "Miniaturized real-time monitoring system for -lactate and glucose using microfabricated multi-enzyme sensors," Biosens. Bioelectron., vol. 15, no. 9-10, pp. 515-522, 2000.

[18] M. Odijk, A. Baumann, W. Lohmann, F. T. G. Van Den Brink, W. Olthuis, U. Karst, and A. van den Berg, "A microfluidic chip for electrochemical conversions in drug metabolism studies," Lab. Chip, vol. 9, pp. 1687-1693, 2009.

[19] K. Gong, Y. Yan, M. Zhang, L. Su, S. Xiong, and L. Mao, "Electrochemistry and electroanalytical applications of carbon nanotubes: A review," Anal. Sci., vol. 21, pp. 1383-1393, 2005.

[20] A. Mayer, N. M. Miskovsky, and P. H. Cutler, "Theoretical comparison between field emission from single-wall and multi-wall carbon nanotubes," Phys. Rev. B, vol. 65, no. 15, p. 155420, 2002.

[21] J. Wang, M. Musameh, and Y. Lin, "Solubilization of carbon nanotubes by nafion toward the preparation of amperometric biosensors," J. Amer. Chem. Soc., vol. 125, no. 9, pp. 2408-2409, 2003.

[22] C. Boero, S. Carrara, G. Del Vecchio, L. Calzá, and G. D. Micheli, "Highly sensitive carbon nanotube-based sensing for lactate and glucose monitoring in cell culture," IEEE Trans. Nanobiosci., vol. 10, no. 1, pp. 59-67, 2011.

[23] W. A. Klee and M. Niremberg, "A neuroblastoma x glioma hybrid cell line with morphine receptors," Proc. Nat. Acad. Sci., vol. 71, pp. 3474-3477, 1974

[24] D. R. Thevenot et al., Electrochemical Biosensors: Recommended Definitions and Classification, Tech. Rep, IUPAC, 1999.

[25] G. Leegsma-Vogt, K. Venema, N. Brouwer, J. B. Gramsbergen, S. Copray, and J. Korf, "Quantitative on-line monitoring of cellular glucose and lactate metabolism in vitro with slow perfusion," Anal. Chem., vol. 76 , no. 18 , pp. $5431-5435,2004$.

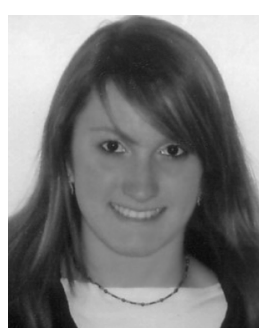

Cristina Boero received the M.Sc. degree in biomedical engineering from Politecnico di Torino, Italy, in 2007. She is currently working toward the Ph.D. degree in the Laboratory of Integrated Systems, École Polytechnique Fédérale de Lausanne (EPFL), Lausanne, Switzerland.

Her main research interest is in electrochemical biosensors for the detection of metabolites in stem cell cultures. The goal is to develop a fully integrate chip to detect such metabolites in real-time into Petri dishes. She is also interested in the functionalization of electrodes with nanostructures, like carbon nanotubes, and the interaction between proteins and nanostructures.

Ms. Boero received the Gold Leaf Award at PRIME in 2009.

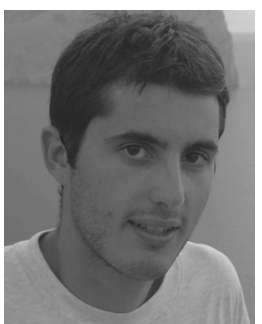

Jacopo Olivo received the M.Sc. degree in electrical engineering from the University of Bologna, Bologna, Italy, in 2008 and is currently working toward the Ph.D. degree at the Integrated System Laboratory, École Polytechnique Fédérale de Lausanne, Lausanne, Switzerland.

During his master's project, he became involved in the field of biosensors concerning the design and development of an integrable system for electrochemical measurements in point-of-care applications for personalized medicine. His research interest focuses on energy scavenging techniques for implantable biosensors.

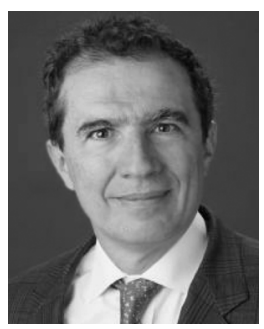

Giovanni De Micheli (F'94) received the nuclear engineer degree from Politecnico di Milano, Italy, in 1979, and the M.Sc. and Ph.D. degrees in electrical engineering and computer science from University of California, Berkeley, in 1983 and 1983, respectively.

$\mathrm{He}$ is Professor and Director of the Institute of Electrical Engineering and of the Integrated Systems Centre at École Polytechnique Fédérale de Lausanne (EPFL), Lausanne, Switzerland. He is Program Leader of the Nano-Tera.ch program. His research interests include emerging technologies, networks on chips and 3-D integration. He is also interested in heterogeneous platform design including electrical components and biosensors, as well as in data processing of biomedical information.

Dr. De Micheli is a Fellow of ACM and a member of the Academia Europaea. He is the recipient of the 2003 IEEE Emanuel Piore Award, Golden JubileeMedal from IEEE CAS Society in 2000, D. Pederson Award in 1987 for the best paper, two Best Paper Awards at the Design Automation Conference, in 1983 and in 1993, and a Best Paper Award at the DATE Conference in 2005.

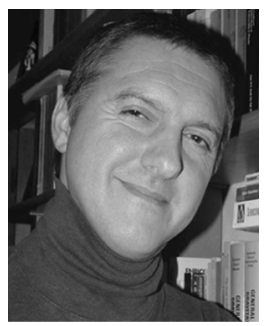

Sandro Carrara received the M.Sc. degree from University of Genoa, Italy, in 1993, and the Ph.D. degree in biochemistry and biophysics from University of Padoa, Italy, in 1997

$\mathrm{He}$ is Senior Scientist and Lecturer at the École Polytechnique Fédérale de Lausanne (EPFL), Lausanne, Switzerland, and Professor of nanobiosensing and micro/nano interfaces at the Department of Electrical Engineering and Biophysics (DIBE), University of Genoa, Italy. He has authored more than 100 publications and 10 patents. He authored Top 25 Hottest Articles (in 2004, 2005, 2008, and 2009) in highly-ranked international journals. His main scientific interest is in electrical phenomena mediated by nanostructured molecular thin films and the development of protein and DNA-based CMOS biochips.

Dr. Carrara is the founder and Editor-in-Chief of BioNanoScience and Associate Editor of the IEEE TRANSACTIONS ON BIOMEDICAL CIRCUITS AND SYSTEMS and of the IEEE SENSORS JOURNAL. 